\title{
1 Host-age prediction from fecal microbiome composition in laboratory mice
}

2 Running title: Microbiome profiling enables prediction of mice age

4 Adrian Low ${ }^{a^{*}}$, Melissa Soh ${ }^{a^{*}}$, Sou Miyake ${ }^{\mathrm{a}}$, Henning Seedorf ${ }^{\mathrm{a}, \mathrm{b}, \#}$

${ }^{a}$ Temasek Life Sciences Laboratory, 1 Research Link, Singapore 117604, Singapore

${ }^{b}$ Department of Biological Sciences, National University of Singapore, Singapore 117604,

$8 \quad$ Singapore

9

$10 \quad{ }^{\#}$ Author for correspondence

$11 *$ These authors contributed equally. Author order was determined alphabetically

12

13 Temasek Lifesciences Laboratory, 1 Research Link, Singapore 117604, Singapore

14 Email: henning@tll.org.sg

\section{ORCID}

16 Adrian Low https://orcid.org/0000-0003-0997-2490

17 Melissa Soh https://orcid.org/0000-0002-7578-9515

18 Sou Miyake https://orcid.org/0000-0001-7507-1653

19 Henning Seedorf https://orcid.org/0000-0002-5763-0236 


\section{ABSTRACT}

25 The life-long relationship between microorganisms and hosts has a profound impact on the overall

26 health and physiology of the holobiont. Changes in microbiome composition throughout the

27 lifespan of a host remain, however, largely understudied. In this study, the fecal microbiome of 28 conventionally raised C57BL/6J mice was analyzed throughout almost the entire expected

29 lifespan, from 'maturing' (9 weeks) until 'very old' age (112 weeks). Analysis of alpha and beta

30 diversity suggests that gradual microbiome changes occur throughout the entire murine life but

31 appear to be more pronounced in 'maturing' to 'middle-aged' phases. Phylum-level analysis

32 indicates a shift in the Firmicutes/Bacteroidetes ratio in favor of the Firmicutes in the second year

33 of adulthood. Varying successional patterns throughout life were observed for many Firmicutes

34 OTUs, while relative abundances of Bacteroidetes OTUs varied primarily in the early life phases.

35 Microbiome configurations at given time points were used as training sets in a Bayesian model,

36 which in turn effectively enabled the prediction of host age. The fecal microbiome composition

37 may therefore serve as an accurate biomarker for aging. This study further suggests that age-

38 associated compositional differences may have considerable implications for the interpretation and

39 comparability of animal model-based microbiome studies.

\section{Importance}

43 The life-long relationship between microorganisms and hosts has a profound impact on the overall

44 physiology of the holobiont. Understanding the extent of gut microbiome compositional changes

45 over the expected mouse lifespan may allow to better understand the interplay of microbiome and

46 the host at the different life stages. In this study, we performed a two-year longitudinal study of

47 murine fecal microbiome. Using fine-scale microbiome profiling we were able to predict the host 
age from the fecal microbiome composition. Moreover, we observed that the rate of compositional change appears to slow with age. The description of the compositional changes in commonly used C57BL/6J mice can be used to optimize selection of age-associated mouse models and highlights

51 the use of microbiome-profiling as biomarker for aging.

53 Keywords: Mouse gut microbiota; 16S rRNA gene amplicon sequencing; aging; temporal analysis; longitudinal analysis; succession; conventionally raised mice

\section{Introduction}

The gut microbiome is known to exert wide-ranging effects on host health (1). As such, understanding the dynamics of the murine gut microbiome is important for mouse model-based research. Studies have shown that humans and mice harbor distinct gut microbiomes at different phases of their lives $(2,3)$. While compositional variability can be attributed to factors such as

61 housing and diet $(4,5)$, age related factors such as host immunity-gut microbiota interactions are

62 more likely to affect gut homeostasis and host health under controlled conditions (6). Over a mouse

63 lifespan, other age-related changes include behavior (7), physiology (8), cellular biochemistry and

64 susceptibility to diseases $(9,10)$. Notwithstanding, most researchers cite practical reasons over

65 host biology when selecting younger mice for research (11).

67 well characterized nor understood thus far (11). Longitudinal studies are rarely performed

68 compared to cross-sectional studies, partly due to the convenience of obtaining mice of different

69 ages and accumulative cost of mice maintenance. Cross-sectional studies of the murine gut

70 microbiome are generally focused on the early or later years of the murine life $(2,12,13)$. One

71 such study examined the gut microbiomes of 'young' (24 weeks old), 'middle age' (84 weeks old) 
72 and 'very old' (122 weeks old) female C57BL/6J mice and observed major shifts in nine of the

73 most abundant bacterial families and functional genes with age and frailty (2). These shifts indicate

74 that life-stage specific microbiome compositions could potentially also serve as biomarker of the

75 host age. Nevertheless, the reproducibility of these shifts remains uncertain due to small sample

76 size and high inter-individual variability, which may result in diverging microbiomes among mice

77 of different batches $(2,4)$.

78 This study aims to elucidate the temporal changes in the gut microbiome of conventionally

79 raised and widely used adult C7BL/6J mice. The longitudinal analysis of the murine gut

80 microbiome over the course of its entire adult lifespan provides a highly resolved compositional

81 profile, indicates life-stage specific microbiome compositions and may allow for more specific

82 selection of mouse models for research questions relevant to the host age.

\section{Results}

\section{Microbiome composition changes throughout life}

The fecal microbiomes of nine-week-old C57BL/6J mice were characterized at regular

87 intervals over 103 weeks (Figure 1a for experimental timeline and life phases and Supplementary

88 Figure S1 for survival curve). Alpha and beta diversities between life phases, as well as individual

89 timepoints, were compared to evaluate if general changes in diversity could be observed

90 throughout the lifespan of the mice. The temporal changes in alpha diversity were compared using

91 Shannon, Simpson, Chaol and Pielou's evenness indices (Figure 1b for life phases and

92 Supplementary Figure 2 for fine scale). Shannon, Simpson's diversity indices and Pielou's

93 evenness index showed similar trends. OTU diversity significantly increased as the mice aged from

94 'maturing'(MR) to 'mature'(MA) (FDR-corrected $P$-values $<0.01$; Wilcoxon test; Supplementary

95 Table S3), while no significant differences were observed between 'MA' to 'middle age'(MD), 
'MD' to 'old'(OD) and 'OD' to 'very old'(VO) (Figure 1b). However, the trend was different for

97 Chaol index, where significant differences (FDR-corrected $P$-values $<0.05$; Wilcoxon test) were

98 observed between 'MA' vs 'MD' and 'MD' vs 'OD' (Figure 1b). Collectively, the indices indicate

99 that species evenness was the primary change between 'MR' to 'MA' and species richness was

100 accountable for the change between ' $\mathrm{MA}$ ' to 'OD'. Comparison of incremental timepoints

101 revealed gradual changes in Shannon and Simpson diversity and Pielou's evenness with significant

102 differences observed between five or fewer pairs of incremental timepoints (e.g. 38 vs 43 weeks,

10347 vs 52 weeks, 52 vs 56 weeks old, 77 vs 82 weeks and 82 vs 86 weeks for Shannon diversity)

104 (Supplementary Figure S2; Supplementary Table S3b). Longitudinal change in Chao1 index

105 between incremental timepoint was indistinguishable between all but one pair of timepoints that

106 showed a significant difference (104 vs 108 weeks) (Supplementary Figure S2).

107 Consistent with this finding, there was a statistically significant difference in beta-diversity

108 between 'maturing' and 'mature' with significant difference (FDR-corrected $P$-values $<0.001$;

109 Permutational multivariate analysis of variance (PERMANOVA)) for Bray-Curtis (Supplementary

110 Table S3c) and weighted-UniFrac (Supplementary Table S3d) compared to later phases as the mice

111 aged (Supplementary Figure S3). The microbiota continued to change compositionally between

112 consecutive phases of life with statistical significance (FDR-corrected $P$-values $<0.001$ for both

113 matrices; PERMANOVA) except between 'OD' and 'VO' (Supplementary Tables S3c,d).

114 Longitudinal comparison at closer timepoints revealed that the gut microbiotas from 56 week-old

115 to 112 week-old mice were more stable, with decreasing number of significantly different

116 microbiota between incremental timepoints (six pairs with FDR-corrected $P$-values $<0.05$ for both

117 matrices; PERMANOVA; Supplementary Tables S3e,f) compared to ages before 56 weeks (Figure

118 1c). Based on Bray-Curtis dissimilarity and weighted-UniFrac distance matrices, there was a 119 decreasing frequency of significantly different pairs of timepoints as the mice aged 
120 (Supplementary Figures S6). Taken together, the results indicate that the murine gut microbiota

121 underwent more compositional changes in the first year compared to the second year.

Differences in microbiome composition could be observed at different taxonomic levels.

123 Figure 2a shows the relative abundance of the major OTUs $(\geq 0.5 \%$ mean relative abundance) of

124 the seven phyla over the 103-week study ( $n=26$ timepoints). Out of 1,092 OTUs, 9.3\% (101

125 OTUs) accounted for the major OTUs. Firmicutes and Bacteroidetes constituted the predominant

126 phyla (combined relative abundance $=96.43 \% \pm 1.03($ mean \pm SD) $)$ over the entire time course,

127 especially from 'MA' phase (17-and 22-week-old mice) to 'MD' (47 weeks old) where the phylum

128 Bacteroidetes was generally more abundant (Figure 2b). Firmicutes started to comprise

129 consistently higher relative abundances than Bacteroidetes from approximately 60 weeks (Figure

130 2b). Similarly, most less abundant phyla ( $\leq 3 \%$ mean relative abundance) were detectable at higher

131 relative abundance in middle-aged and older mice (see Supplementary Information for details and

132 Supplementary Figure S4).

OTU-level analysis revealed that only $20 \%$ (22 of the 110 OTUs) of the most abundant

134 OTUs were detected at all analyzed timepoints (Figure 3; Supplementary Table S1). Their relative

135 abundances differed across life stages. Four OTUs that were among the more abundant ones during

136 the 'MR' stage, namely OTU0133 (closest BLAST hit (\% 16S rRNA gene identity):

137 Paramuribaculum intestinale B1404 (92.6\%); 24.2\% relative abundance), OTU0905 (Turicibacter

138 sp. TS-3 (100\%); 6.8\% relative abundance), OTU0255 (Lactobacillus johnsonii G2A (99.4\%);

$1396.6 \%$ relative abundance) and OTU0727 (Anaerotignum sp. 1XD42-85 (99.4\%); 1.2\% relative

140 abundance), but all decreased to approximately $1 \%$ relative abundance at 'MA' until 'very old

141 (VO)’ stage (Supplementary Table S1). OTU0255 (Lactobacillus johnsonii G2A (99.4\%)) was

142 solely responsible for changes to Lactobacillaceae family over time. OTU0610 (Faecalibaculum

143 rodentium Alo17 (99.4\%)) stood out as the predominant OTU for the majority of adulthood with 
144 mean relative abundances of $15.5 \%$ to $17.9 \%$ between 'MR' to 'VO' phase (Supplementary Table

145 S1). The remaining 18 OTUs observed at all timepoints stayed relatively constant throughout the 146 mouse adult lifespan.

147 Successional patterns of OTUs within different phyla could be observed. The majority of 148 Bacteroidetes OTUs were only detectable from the beginning of the 'MA' phase and were largely 149 present until the mice were 112 weeks old (Figure 2a). This is in contrast with the majority of 150 Firmicutes OTUs that were detectable at 9 weeks but have different succession patterns, e.g. a few 151 OTUs decreased to low relative abundances as the mice aged (Figure 2a). Actinobacteria, 152 represented by two distinct OTUs had similar succession patterns to the majority of Bacteroidetes 153 OTUs (Figure 2a). Cyanobacteria, represented by OTU0941 (Candidatus Melainabacteria 154 bacterium MEL.A1 (96.4\%)), were detected at 52 weeks and remained at similar levels until 112 155 weeks (Figure 2a). Proteobacteria, represented by two OTUs showed dissimilar succession 156 patterns with OTU0928 (Parasutterella sp. Isolate_NM82_D38 (99.4\%)) detected later in the

157 'MD' phase compared to OTU0236 (Desulfovibrio sp. 16x (99.4\%)) detected earlier at 'MA' 158 phase. Tenericutes, represented by a single long-term observable bacterial OTU0354 159 (Anaeroplasma abactoclasticum 6-1 (92.1\%)), had lowest mean relative abundance $(0.07 \%)$ at 160 'MD' compared to other phases ranging from 0.23\%-0.88\% (Figure 2c; Supplementary Table S1).

\section{Identification of OTUs predictive for host age}

The observation of the successional OTUs prompted the development of a random forest

164 regression model to identify OTUs predictive of the 26 analyzed timepoints (14). This model 165 showed a strong correlation $(r=0.958 ; P<0.001)$ between predicted and actual microbiota for 166 each timepoint. Of the 100 predictive OTUs, just five OTUs (OTU0928, OTU0126, OTU0330, 167 OTU0111 and OTU0762) have a cumulative importance score of $86 \%$, indicating that these OTUs 
168 have the greatest effect on the regression model (Supplementary Table S2a). Furthermore, 28

169 predictive OTUs of low abundance were identified (Supplementary Figure S5), of which two

170 OTUs, OTU0232 (closest BLAST hit (\% 16S rRNA gene identity): Adlercreutzia sp. D16-63

$171(95.5 \%))$ and OTU0708 (Acholeplasmatales bacterium oral (91.8\%)) were detectable at all

172 timepoints (Supplementary Table S2b). The mean relative abundances of the top three to five

173 OTUs of each phylum ranked by importance score over time are shown in Figure 2c. Predictive

174 Firmicutes OTUs varied in their successional patterns: OTU0111 (Robinsoniella sp. D2-1X-13

$175(95.5 \%))$ was more pronounced in earlier stages but diminished during 'OD and 'VO' stages, while

176 in contrast, three OTUs (OTU0844 (Clostridium cocleatum (100\%)), OTU0757 (Syntrophococcus

177 sp. BS-2 (92.6\%)) and OTU0335 (Lactobacillus murinus V10 (99.4\%)) were mid to late

178 successors, and OTU0762 (Intestinimonas butyriciproducens DSM 104946 (95.9\%)) was only

179 detectable during the late phases (Figure 2c). Of the Bacteroidetes OTUs, three OTUs (OTU1010

180 (Paramuribaculum intestinale (99.4\%)), OTU0705 (Muribaculum sp. J10 (96.0\%)) and OTU0782

181 (Muribaculum sp. 26x (99.4\%)) became detectable from 'MA' phase and remained relatively

182 similar throughout (Figure 2c). The predictive Actinobacteria OTUs had a similar succession

183 pattern, becoming detectable from 12 weeks old but with different relative abundances (Figure 2c).

184 Among the Proteobacteria OTUs, OTU0236 (Desulfovibrio sp. 16x (99.4\%)) was an early

185 successor, OTU0928 (Parasutterella sp. Isolate_NM82_D38 (99.4\%)) was a mid-successor, and

186 OTU0815 (Bilophila wadsworthia Marseille-AA00033 (94.9\%)) and OTU0527 (Dongia sp. CON-

$18765(93.4 \%)$ ) were late successors (Figure 2c). The two Cyanobacteria OTUs (OTU0941 and

188 OTU0330) have closest 16S rRNA gene identity (96.4\% and 92.4\%, respectively) to Candidatus

189 Melainabacteria bacterium MEL.A1 were mid-successors (Figure 2c). Two Tenericutes OTUs

190 present from the beginning decreased to low relative abundance between the 'mature' and 'middle 
191 age' phases, before OTU0354 (Anaeroplasma abactoclasticum 6-1 (92.1\%)) increased again in

192 relative abundance at 'old' phase (Figure 2c).

194 Prediction of host age based on fecal microbiome composition

The observed successional pattern during the aging process and the identification of

196 predictive OTUs for specific timepoints prompted the question: can the animal age be inferred

197 from fecal microbiome composition? For this purpose the SourceTracker software, originally

198 designed to use Bayesian statistics to determine the contribution of 'source' communities to 'sink'

199 communities for monitoring water quality, was applied to our longitudinal study (15). Selection of

200 samples $(n=7)$ as 'source' communities representative of the five life phases was based on

201 timepoints with the fewest number of significantly different microbiomes as guided by pair-wise

202 permutational multivariate analysis of variance (PERMANOVA) tests of Bray-Curtis

203 dissimilarities (Supplementary Figure S6). The analysis revealed that specific life phases can in

204 principle be predicted from fecal microbiomes (Figure 4a). An approximate age prediction for

205 individual timepoints was performed by using the sum of the products of predicted life stage

206 fractions and midpoints of life stages:

$$
\mathrm{y}_{\mathrm{age}}=\mathrm{aMR}_{\mathrm{MR}}+\mathrm{aMA}_{\mathrm{MA}}+\mathrm{a}_{\mathrm{MD}} \mathrm{XMD}_{\mathrm{MD}}+\mathrm{aOD}_{\mathrm{OOD}}+\mathrm{aVD}_{\mathrm{V}} \mathrm{XV}_{\mathrm{V}}
$$

208 where yage is the predicted age of the mouse based on fecal microbiome composition; a is the

209 midpoint of the life stage in weeks old (i.e., 'MR' $=10.5$, 'MA' $=19.5$, 'MD' $=51.5$, 'OD' = 93

210 and ' $\mathrm{VO}$ ' $=110$ ); and $\mathrm{x}$ is the predicted proportion of the life stage. The prediction was found to

211 be highly accurate for younger animals (9-47 weeks old Spearman $\rho=0.925)$. As expected, the

212 level of accuracy decreased for animals that are older $(52-112$ weeks old Spearman $\rho=0.774)$

213 (Figure 4b). This is in line with the PERMANOVA analysis that showed that the microbiome is

214 becoming more stable in late stages of adulthood (Supplementary Figure S6). 


\section{Discussion}

217 It has been well established that the microbiomes of humans and animals change significantly in 218 early life phases, e.g. after the weaning transition from milk to solid foods $(12,16,17)$, but the

219 longitudinal changes of the gut microbiome throughout adulthood are less well characterized. In

220 this study, we characterized the gut microbiome of conventionally raised adult male C57BL/6J

221 mice at regular fine-scale intervals for two years, which corresponds to the approximate lifespan

222 of this species (10). We observed microbiome variations during murine gut development not

223 reported in previous cross-sectional or shorter longitudinal murine studies $(2,18,19)$, such as a

224 significant increase in species richness and evenness during the early stages between 'MR' and

225 'MA' phase where shifts in abundance between phyla were observed (Figure 2a), but also in the

226 later phases. Interestingly, a similar increase in alpha-diversity of swine gut microbiome was also

227 observed during the growing years $(20,21)$, indicating that it may be an evolutionary trait that is

228 conserved across species. The increased diversity also shows that the adult gut microbiome

229 continues to change significantly after reaching host maturity between 12 to 17 weeks old. This

230 has implications for research using mouse models. A recent meta-analysis showed that the vast

231 majority of mice used for experiments is aged between 8-12 weeks old (11). In view of the current

232 study, mice age should be selected carefully based on the research question.

Another notable observation is that the rate of change did not appear to be constant. There

234 was a higher frequency of compositional change to the gut microbiome in the first year, while the

235 second year is marked by a reduced frequency of compositional change, especially at the lower

236 taxonomic levels (i.e. fewer OTUs in succession and more OTUs in decline) from 'OD' to 'VO'

237 phase. At this stage, the host may experience a decreased rate of glucose and fatty acid metabolism 238 and reduced energy expenditure (22). Feed intake (normalized to bodyweight) has been shown to 
not differ significantly in mice from mature to very old age (23), which indicates that the feed

240 intake has only limited effect as underlying cause for the observed changes. This could explain the

241 absence of significant difference in alpha-diversity between 'OD' and 'VO' mice (Figure 1). While

242 it is generally agreed that a loss of diversity at old age may result in undesirable phenotypes such

243 as inflammation or frailty in mice $(2,24)$ and increased frailty and reduced cognitive performance

244 in humans $(25,26)$, our study suggests that loss of gut diversity may not necessary be associated

245 with chronological age under controlled conditions.

Our analyses have revealed similar (e.g. Actinobacteria and Bacteroidetes) and variable

247 (e.g. among Firmicutes OTUs) succession patterns as well as consistently observable taxa among

248 the different phylogenetic lineages (Supplementary Tables S1 and S2b). Firmicutes remained the

249 predominant phylum throughout 'OD' and 'VO' phases. However, prior to the late stage shift in

250 relative abundance, Bacteroidetes relative abundance was higher than that of Firmicutes (Figure

251 2b). This observation in mice is in contrast with previous studies on elderly humans that generally

252 reported an age-related decrease in the Firmicutes to Bacteroidetes ratio $(27,28)$. It is currently

253 not possible to determine if these divergent patterns are specific to the host species, as has been

254 shown for other factors such as immune maturation or microbe-host interaction (29, 30), or

255 whether it may potentially be an artefact, e.g. resulting from cross-sectional analyses. Comparisons

256 of our data to other murine studies may be hampered, since compositional differences may be

257 influenced by many factors including housing facilities (4).

258 In our study, Firmicutes was the phylogenetically most diverse phylum, with the majority

259 of OTUs detectable from the beginning. Twenty of the 24 identified long-term observable OTUs

260 including two of the predictive OTUs, belong to the Firmicutes. Among them are OTUs of

261 Lactobacillus that are thought to be associated with a healthy gut (31). In particular, L. johnsonii

262 isolated from $\mathrm{BALB} / \mathrm{c}$ mice has been shown to protect the host by influencing the host immune 
response against airborne allergens and viral infection (32). In our study, the proportional decrease

264 in L. johnsonii (OTU0255) at 'OD' and 'VO' life stage is consistent with previous studies of aged

265 mice (141 weeks old) and humans (76 to 100 years old) and may indicate a reduced protective

266 effect from this species $(18,33)$. Among the few OTUs (e.g. Corynebacterium (OTU0822)) that

267 emerged in the late phases of murine life (Supplementary Table S1), Corynebacterium spp. have

268 been shown to also increase in relative abundance with age in humans from 79 years old and above

269 (34). Hence, 'OD' and 'VO' C57BL/6J mice might be suitable models for studies related to

270 infections from potentially pathogenic Corynebacterium spp. (35). Faecalibaculum rodentium

271 Alo17, previously isolated from a 36-week old female C57BL/6J mouse, was shown here to

272 represent the most abundant OTU in adult mice (Figure 3) (36). Altogether, these results suggest

273 that the microbiome gradually changes throughout the lifespan of the animals and that OTUs of

274 different phyla are affected to a varying degree, which in turn results in characteristic life-stage

275 dependent microbiome configurations.

The random forest regression model identified key OTUs, including some low-abundance

277 OTUs, that are predictive of host age (Figure 2c, Supplementary Figure S5 and Supplementary

278 Table S2). Specifically, OTU0928 (Parasutterella sp. of Proteobacteria) was most predictive for

279 the temporal changes with succession at 'MD' stage of the mouse life (Figure 2c). Parasutterella

280 is ubiquitous in the gut microbiomes of mammalian and human hosts and may benefit the host

281 with bile acid maintenance and cholesterol metabolism (37). The successional pattern observed for

282 most Proteobacteria OTUs to be mid to late successors is consistent with other murine and human

283 gut microbiome studies $(2,18,27,38)$. In contrast, Desulfovibrio sp. 16x (OTU0236), a sulfate

284 reducing bacterium, represented an exception to the Proteobacteria successional pattern: This OTU

285 was detected from 'MA' to 'VO' stages (Figure 2c), supposedly performing an important role

286 along with acetogens in lowering hydrogen partial pressure in the gut $(39,40)$. Notably we did not 
detect any Enterobacteriaceae, however, this may be due to the commercial source of the laboratory mice in this study (41).

Our study clearly demonstrates the continuous changes of the gut microbiome and the 290 different OTU succession patterns. The gut microbiomes of C57BL/6J mice largely followed host 291 physiological development, but the periods of transition varied (10). For example, the transition 292 from 'MA' to 'MD' was much longer (21 weeks) than the transition from 'MD' to 'OD' (5 weeks). 293 The reliable host age prediction showed that there was sufficient distinction among few 'source' 294 microbiota to delineate the majority of fecal microbiomes from 'MR' to 'OD'. Limits to the 295 prediction were observed for mice between the 'OD' and 'VO' stage, where the microbiota were too similar to be differentiated. The natural lifespan of mice sets limits to extend these experiments 297 and to observe more small-scale dissimilarities between microbiomes of the very late stages. It 298 also needs to be noted that these experiments were conducted in a well-controlled laboratory 299 environment and the same standard laboratory feed was used for the entire duration. Using a 300 different diet, alternating between diets, or performing other experimental modifications may also 301 have lasting effects on the microbiome composition and its association with age as previously 302 reported (18). Nevertheless, this study highlights the age-associated natural variation of the gut 303 microbiome of conventionally raised C57BL/6J mice.

In summary, this study shows that the fecal microbiome of laboratory mice changes 305 substantially throughout the entire adult age. Consequentially, this has implications for the design 306 of experiments where the microbiome can be considered a contributing factor affecting host 307 physiology. Furthermore, this study highlights that the microbiome can serve as a biomarker of 308 aging and that host age can be inferred from microbiome composition. 
Materials and methods

\section{Animal husbandry, fecal sampling and DNA extraction}

312 Experiments involving mice were approved by the Institutional Animal Care and Use Committee

313 (IACUC number: TLL-17-018) in accordance with National Advisory Committee for Laboratory

314 Animal Research guidelines and were performed at Temasek Life Sciences Laboratory, Singapore

315 with supervision by trained veterinarians. Male C57BL/6J mice were purchased from InVivos

316 (Singapore) at 63 days of age. The mice $(n=20)$ were kept in four cages of five mice each and

317 maintained on standard chow (carbohydrate $=62.3 \%$, protein $=25.5 \%$, fat $=13.1 \% ;$ PicoLab $^{\circledR}$

318 Rodent Diet 20; LabDiet, St Louis. MO, USA) ad libitum. Fecal materials were taken from all

319 mice at one (10 weeks old) and two-week (12 weeks old) intervals followed by monthly (4-5 weeks

320 old) intervals from 17 to 112 weeks old except upon arrival (9 weeks old) where a sub-sample of

321 mice $(n=4)$ were sampled (Figure 1). Fecal matter was collected fresh in $2 \mathrm{~mL}$ sterile screw-

322 capped tubes and flash-frozen in liquid nitrogen before storing at $-80{ }^{\circ} \mathrm{C}$ until DNA extraction. A

323 bead-beating phenol chloroform DNA extraction method was used on all fecal samples as

324 previously described (42).

325

Amplicon sequencing of 16S rRNA genes

A dual indexed 16S rRNA gene amplicon library was generated using primers 515F (43) and 806R (44) in triplicate PCRs per sample according to the protocol and indexes described in

329 the Earth Microbiome Project (45). Illumina MiSeq sequencing was performed at the Genome

330 Institute of Singapore according to the MiSeq reagent kit v2 $(2 \times 250 \mathrm{bp})$ preparation guide 331 (Illumina, San Diego, CA, USA). 
All MiSeq fastq files were deposited in the NCBI Sequence Read Archive under the Project ID PRJNA503299. The fastq files were processed using QIIME 2 version 2020.2 (accessed on 15 February 2020) using “qiime tools import" (46). Default options were used for all QIIME 2 scripts unless stated otherwise. Forward reads were quality denoised, trimmed, de-novo clustered and chimera checked using the "qiime dada2 denoise-single" command for DADA2 (47) with the following options: “--p-trim-left 23”, which removes the first 23 bp of reads and "--p-trunc-len 199”, which truncates at $199 \mathrm{bp}$. The quality filtered reads of $176 \mathrm{bp}(n=433)$ have a median count of 13,596 reads. To reduce the number of highly similar sequences, denoised single-end 342 reads were clustered at 99\% similarity OTUs using the "qiime vsearch cluster-features-de-novo" 343 command with “--p-perc-identity 0.99” option, (48) which generated 1,107 OTUs. A single OTU

344 for chloroplast was filtered prior to further analysis. Alignment was performed using the "qiime 345 phylogeny align-to-tree-mafft-fasttree" command. The command "qiime diversity core-metrics346 phylogenetic" with the option “--p-sampling-depth 3422" was used to generate alpha and beta 347 diversity measures including Bray-Curtis dissimilarity, weighted- and unweighted-UniFrac 348 matrices, PCoA plots and a rarefied OTU table, which were visualized as Emperor plots (49).

349 Custom PCoA plots were generated using the "qiime emperor plot" command to plot samples 350 grouped by age on the $\mathrm{x}$-axis against the first principal coordinate (PC1) on the $\mathrm{y}$-axis. Alpha351 diversity, relative abundance and heatmap plots were generated using the phyloseq (50), ggplot2 352 (51), reshape (52), microbiome (53), genefilter (54), data.table (55) and patchwork (56) packages 353 for $\mathrm{R}$ (57). Taxonomic identities were assigned to OTUs using the "qiime feature-classifier 354 classify-sklearn” command against a trained classifier SILVA SSU for V4 region version 132 non355 redundant $99 \%$ identity database (58). To obtain updated taxonomic identities not curated in 356 SILVA version 132, OTUs were annotated to the GenBank non-redundant database (accessed 28 357 Feb 2020) using the megablast function of BLASTn version 2.10.0+ (59, 60). To identify OTUs 
that are predictive of the temporal changes, the "qiime longitudinal feature-volatility" command was used with “--p-n-estimators 100" and “--p-random-state 10" options that adopts the random forest regressor as a machine learning method $(14,61)$. SourceTracker version 0.9.1 was used to estimate the mouse age using the rarefied table (15). As SourceTracker is estimating the probability

362 of a 'sink' microbiota compared to a "source" microbiota, samples picked as 'source' were based

363 on the timepoints with the fewest number of significantly different pairs i.e. more similar

364 microbiome to most samples using the pair-wise permutational analysis of variance

365 (PERMANOVA) analysis of the Bray-Curtis matrix (Supplementary Figure S6). One 366 representative timepoint was picked as "source" for each of the phases with three or fewer 367 timepoints namely, 'MR', 'MA' and 'VO'. If all timepoints within each phase shared the same 368 number of significantly different pairs, the median point was selected. Two timepoints were 369 selected as "source" for the other two phases. All samples including those selected as "source" 370 were also analyzed as "sinks".

\section{Statistical analysis}

The Kruskal-Wallis and pairwise Wilcoxon rank sum tests were performed using phyloseq

374 (50), tidyr (62) and dplyr (63) packages for R (57). Pair-wise PERMANOVA tests were performed 375 using the "qiime diversity beta-group-significance" QIIME 2 command based on 9,999 376 permutations with $P$-values corrected using the Benjamini-Hochberg FDR method (64). Spearman 377 correlations and polynomial regression for SoureTracker prediction between actual and predicted 378 ages were performed using R (57). $P$-value $<0.05$ is considered statistically significant. 
381 We thank and Muhammad Khairillah Bin Nanwi at TLL biocomputing for bioinformatics support.

382 We also thank Subramanian Kabilan and Adeline Wong for support with animal maintenance and

383 sample collection, respectively.

384

385 Funding details

386 This work was supported by Temasek Life Sciences Laboratory.

387

388 Competing Interests

389 None to declare.

390

\section{REFERENCES}

392 1. Kundu P, Blacher E, Elinav E, Pettersson S. 2017. Our Gut Microbiome: The Evolving 393 Inner Self. Cell 171:1481-1493.

394 2. Langille MG, Meehan CJ, Koenig JE, Dhanani AS, Rose RA, Howlett SE, Beiko RG. 2014.

395 Microbial shifts in the aging mouse gut. Microbiome 2:50.

396 3. Mariat D, Firmesse O, Levenez F, Guimaraes V, Sokol H, Dore J, Corthier G, Furet JP. 397 2009. The Firmicutes/Bacteroidetes ratio of the human microbiota changes with age. BMC $398 \quad$ Microbiol 9:123.

399 4. Rausch P, Basic M, Batra A, Bischoff SC, Blaut M, Clavel T, Glasner J, Gopalakrishnan 400 S, Grassl GA, Gunther C, Haller D, Hirose M, Ibrahim S, Loh G, Mattner J, Nagel S, Pabst 401 O, Schmidt F, Siegmund B, Strowig T, Volynets V, Wirtz S, Zeissig S, Zeissig Y, Bleich 402 A, Baines JF. 2016. Analysis of factors contributing to variation in the C57BL/6J fecal microbiota across German animal facilities. Int J Med Microbiol 306:343-355. 
404 5. Myles IA, Fontecilla NM, Janelsins BM, Vithayathil PJ, Segre JA, Datta SK. 2013.

405 Parental dietary fat intake alters offspring microbiome and immunity. J Immunol

$406 \quad 191: 3200-9$

407 6. Belkaid Y, Hand TW. 2014. Role of the microbiota in immunity and inflammation. Cell $408 \quad 157: 121-41$.

409 7. Shoji H, Takao K, Hattori S, Miyakawa T. 2016. Age-related changes in behavior in $410 \quad$ C57BL/6J mice from young adulthood to middle age. Mol Brain 9:11.

411 8. Dutta S, Sengupta P. 2016. Men and mice: Relating their ages. Life Sci 152:244-8.

412 9. Childs BG, Durik M, Baker DJ, van Deursen JM. 2015. Cellular senescence in aging and 413 age-related disease: from mechanisms to therapy. Nat Med 21:1424-35.

414 10. Flurkey K, Currer JM, Harrison D. 2007. Mouse models in aging research. In (ed), The 415 mouse in biomedical research. Elsevier, doi:10.1016/B978-012369454-6/50074-1. https://doi.org/10.1016/B978-012369454-6/50074-1.

417 11. Jackson SJ, Andrews N, Ball D, Bellantuono I, Gray J, Hachoumi L, Holmes A, Latcham 418 J, Petrie A, Potter P. 2017. Does age matter? The impact of rodent age on study outcomes. $419 \quad$ Lab Anim 51:160-169.

420 12. Hughes KR, Schofield Z, Dalby MJ, Caim S, Chalklen L, Bernuzzi F, Alcon-Giner C, Le Gall G, Watson AJM, Hall LJ. 2020. The early life microbiota protects neonatal mice from pathological small intestinal epithelial cell shedding. FASEB J doi:10.1096/fj.202000042R.

424 13. Fransen F, van Beek AA, Borghuis T, Aidy SE, Hugenholtz F, van der Gaast-de Jongh C, 425 Savelkoul HFJ, De Jonge MI, Boekschoten MV, Smidt H, Faas MM, de Vos P. 2017. Aged gut microbiota contributes to systemical inflammaging after transfer to germ-free mice. Front Immunol 8:1385. 
14. Breiman L. 2001. Random Forests. Mach Learn 45:5-32.

429 15. Knights D, Kuczynski J, Charlson ES, Zaneveld J, Mozer MC, Collman RG, Bushman FD, Knight R, Kelley ST. 2011. Bayesian community-wide culture-independent microbial

16. Yatsunenko T, Rey FE, Manary MJ, Trehan I, Dominguez-Bello MG, Contreras M, Magris

17. Stewart CJ, Ajami NJ, O'Brien JL, Hutchinson DS, Smith DP, Wong MC, Ross MC, Lloyd

18. Zhang C, Li S, Yang L, Huang P, Li W, Wang S, Zhao G, Zhang M, Pang X, Yan Z, Liu Hyoty H, Vehik K, Krischer JP, Petrosino JF. 2018. Temporal development of the gut

19. Wang J, Lang T, Shen J, Dai J, Tian L, Wang X. 2019. Core gut bacteria analysis of healthy

20. Ke S, Fang S, He M, Huang X, Yang H, Yang B, Chen C, Huang L. 2019. Age-based mice. Nat Commun 4:2163. reveals stage and growth performance associated bacteria. Microbiome 7:109. 
452 22. Houtkooper RH, Argmann C, Houten SM, Canto C, Jeninga EH, Andreux PA, Thomas C, 453 Doenlen R, Schoonjans K, Auwerx J. 2011. The metabolic footprint of aging in mice. Sci $454 \quad$ Rep 1:134.

455 23. Starr ME, Saito H. 2012. Age-related increase in food spilling by laboratory mice may lead to significant overestimation of actual food consumption: implications for studies on dietary restriction, metabolism, and dose calculations. J Gerontol A Biol Sci Med Sci

24. Thevaranjan N, Puchta A, Schulz C, Naidoo A, Szamosi JC, Verschoor CP, Loukov D, Schenck LP, Jury J, Foley KP, Schertzer JD, Larche MJ, Davidson DJ, Verdu EF, Surette MG, Bowdish DME. 2017. Age-associated microbial dysbiosis promotes Intestinal permeability, systemic inflammation, and macrophage dysfunction. Cell Host Microbe

25. O'Toole PW, Jeffery IB. 2015. Gut microbiota and aging. Science 350:1214-1215.

465 26. Jeffery IB, Lynch DB, O'Toole PW. 2016. Composition and temporal stability of the gut microbiota in older persons. ISME J 10:170-82.

467 27. Odamaki T, Kato K, Sugahara H, Hashikura N, Takahashi S, Xiao JZ, Abe F, Osawa R. 2016. Age-related changes in gut microbiota composition from newborn to centenarian: a cross-sectional study. BMC Microbiol 16:90.

470 28. Claesson MJ, Jeffery IB, Conde S, Power SE, O'Connor EM, Cusack S, Harris HM, Coakley M, Lakshminarayanan B, O'Sullivan O, Fitzgerald GF, Deane J, O'Connor M, Harnedy N, O'Connor K, O'Mahony D, van Sinderen D, Wallace M, Brennan L, Stanton C, Marchesi JR, Fitzgerald AP, Shanahan F, Hill C, Ross RP, O'Toole PW. 2012. Gut microbiota composition correlates with diet and health in the elderly. Nature 488:178-84. 
29. Chung H, Pamp SJ, Hill JA, Surana NK, Edelman SM, Troy EB, Reading NC, Villablanca 149:1578-93.

30. Seedorf H, Griffin NW, Ridaura VK, Reyes A, Cheng J, Rey FE, Smith MI, Simon GM,

31. Zmora N, Suez J, Elinav E. 2019. You are what you eat: diet, health and the gut microbiota. Nat Rev Gastroenterol Hepatol 16:35-56.

32. Fujimura KE, Demoor T, Rauch M, Faruqi AA, Jang S, Johnson CC, Boushey HA, Zoratti Lactobacillus enrichment and airway immune defense against allergens and virus infection. Proc Natl Acad Sci U S A 111:805-10.

33. van Tongeren SP, Slaets JP, Harmsen HJ, Welling GW. 2005. Fecal microbiota composition and frailty. Appl Environ Microbiol 71:6438-6442.

491 34. Xu C, Zhu H, Qiu P. 2019. Aging progression of human gut microbiota. BMC Microbiol 19:236.

493 35. Oliveira A, Oliveira LC, Aburjaile F, Benevides L, Tiwari S, Jamal SB, Silva A, Figueiredo HCP, Ghosh P, Portela RW, De Carvalho Azevedo VA, Wattam AR. 2017. Insight of Species. Front Microbiol 8:1937. 
36. Chang DH, Rhee MS, Ahn S, Bang BH, Oh JE, Lee HK, Kim BC. 2015. Faecalibaculum rodentium gen. nov., sp. nov., isolated from the faeces of a laboratory mouse. Antonie Van Leeuwenhoek 108:1309-1318.

37. Ju T, Kong JY, Stothard P, Willing BP. 2019. Defining the role of Parasutterella, a previously uncharacterized member of the core gut microbiota. ISME J 13:1520-1534.

502 38. Biagi E, Nylund L, Candela M, Ostan R, Bucci L, Pini E, Nikkïla J, Monti D, Satokari R, Franceschi C. 2010. Through ageing, and beyond: gut microbiota and inflammatory status in seniors and centenarians. PloS one 5.

39. Rey FE, Faith JJ, Bain J, Muehlbauer MJ, Stevens RD, Newgard CB, Gordon JI. 2010. Dissecting the in vivo metabolic potential of two human gut acetogens. J Biol Chem 285:22082-90.

40. Wolin MJ, Miller TL. Interactions of microbial populations in cellulose fermentation, $\mathrm{p}$ 109-113. In (ed),

41. Velazquez EM, Nguyen H, Heasley KT, Saechao CH, Gil LM, Rogers AWL, Miller BM,

514 42. Rius AG, Kittelmann S, Macdonald KA, Waghorn GC, Janssen PH, Sikkema E. 2012. Nitrogen metabolism and rumen microbial enumeration in lactating cows with divergent residual feed intake fed high-digestibility pasture. J Dairy Sci 95:5024-5034.

517 43. Parada AE, Needham DM, Fuhrman JA. 2016. Every base matters: assessing small subunit rRNA primers for marine microbiomes with mock communities, time series and global field samples. Environ Microbiol 18:1403-14. 
44. Apprill A, McNally S, Parsons R, Weber L. 2015. Minor revision to V4 region SSU rRNA

521

522

523

524

525

526

527

528

529

530

531

532

533

534

535

536

537

538

539

540

541

542

543

806R gene primer greatly increases detection of SAR11 bacterioplankton. Aquat Microb Ecol 75:129-137.

45. Thompson LR, Sanders JG, McDonald D, Amir A, Ladau J, Locey KJ, Prill RJ, Tripathi A, Gibbons SM, Ackermann G, Navas-Molina JA, Janssen S, Kopylova E, Vazquez-Baeza Y, Gonzalez A, Morton JT, Mirarab S, Zech Xu Z, Jiang L, Haroon MF, Kanbar J, Zhu Q, Jin Song S, Kosciolek T, Bokulich NA, Lefler J, Brislawn CJ, Humphrey G, Owens SM, Hampton-Marcell J, Berg-Lyons D, McKenzie V, Fierer N, Fuhrman JA, Clauset A, Stevens RL, Shade A, Pollard KS, Goodwin KD, Jansson JK, Gilbert JA, Knight R, Earth Microbiome Project C. 2017. A communal catalogue reveals Earth's multiscale microbial diversity. Nature 551:457-463.

46. Bolyen E, Rideout JR, Dillon MR, Bokulich NA, Abnet CC, Al-Ghalith GA, Alexander H, Alm EJ, Arumugam M, Asnicar F, Bai Y, Bisanz JE, Bittinger K, Brejnrod A, Brislawn CJ, Brown CT, Callahan BJ, Caraballo-Rodríguez AM, Chase J, Cope EK, Da Silva R, Diener C, Dorrestein PC, Douglas GM, Durall DM, Duvallet C, Edwardson CF, Ernst M, Estaki M, Fouquier J, Gauglitz JM, Gibbons SM, Gibson DL, Gonzalez A, Gorlick K, Guo J, Hillmann B, Holmes S, Holste H, Huttenhower C, Huttley GA, Janssen S, Jarmusch AK, Jiang L, Kaehler BD, Kang KB, Keefe CR, Keim P, Kelley ST, Knights D, et al. 2019. Reproducible, interactive, scalable and extensible microbiome data science using QIIME 2. Nat Biotechnol 37:852-857.

47. Callahan BJ, McMurdie PJ, Rosen MJ, Han AW, Johnson AJ, Holmes SP. 2016. DADA2: High-resolution sample inference from Illumina amplicon data. Nat Methods 13:581-3.

48. Rognes T, Flouri T, Nichols B, Quince C, Mahe F. 2016. VSEARCH: a versatile open source tool for metagenomics. PeerJ 4:e2584. 
544 49. Vazquez-Baeza Y, Gonzalez A, Smarr L, McDonald D, Morton JT, Navas-Molina JA, 545 Knight R. 2017. Bringing the dynamic microbiome to life with animations. Cell Host $546 \quad$ Microbe 21:7-10.

547 50. McMurdie PJ, Holmes S. 2013. phyloseq: an R package for reproducible interactive analysis and graphics of microbiome census data. PloS one 8:e61217.

549 51. Wickham H. 2016. ggplot2: Elegant graphics for data analysis doi:10.1007/978-3-31924277-4. Springer, New York.

551 52. Wickham H. 2007. Reshaping data with the reshape package. J Stat Softw 21:1-20.

552 53. Lahti L, Shetty S. 2012. microbiome R package, http://microbiome.github.io/microbiome.

553 54. Gentleman R, Carey V, Huber W, Hahne F. 2019. Genefilter: Methods for filtering genes from high-throughput experiments, https://bioconductor.org/packages/release/bioc/html/genefilter.html.

556 55. Dowle M, Srinivasan A, Goreck iJ, Chirico M, Stetsenko P, Short T, Lianoglou S, Antonyan E. 2019. data.table: Extension of 'data.frame', https://github.com/Rdatatable/data.table.

559 56. Pedersen TL. 2019. patchwork: The Composer of Plots, v1.01. https://github.com/thomasp85/patchwork.

561 57. R Core Team. 2013. R: A language and environment for statistical computing, R Foundation for Statistical Computing, Vienna, Austria. http://www.R-project.org.

563 58. Quast C, Pruesse E, Yilmaz P, Gerken J, Schweer T, Yarza P, Peplies J, Glockner FO. 564 2013. The SILVA ribosomal RNA gene database project: improved data processing and web-based tools. Nucleic Acids Res 41:D590-6.

566 59. Morgulis A, Coulouris G, Raytselis Y, Madden TL, Agarwala R, Schaffer AA. 2008. 
568 60. Zhang Z, Schwartz S, Wagner L, Miller W. 2000. A greedy algorithm for aligning DNA sequences. J Comput Biol 7:203-14.

570 61. Bokulich NA, Dillon MR, Zhang Y, Rideout JR, Bolyen E, Li H, Albert PS, Caporaso JG. 2018. q2-longitudinal: Longitudinal and paired-sample analyses of microbiome data. mSystems 3. project.org/web/packages/tidyr/index.html.

63. Hadley W, François R, Henry L, Müller K. 2019. dplyr: A Grammar of Data Manipulation. R package version 0.8.0.1, https://CRAN.R-project.org/package=dplyr.

64. Anderson MJ. 2001. A new method for non-parametric multivariate analysis of variance. Austral Ecol 26:32-46.

\section{Figure Legends}

Figure 1. Schematic outline, changes to alpha- and beta-diversities of adult mice as they aged from as C57BL/6J mice were 9 weeks old and ending at 112 weeks old. Life phases of mice and human equivalent age (year) based on previous reports are shown [8,10]. Boxplots of Shannon (b), Simpson's (c), Chaol (d) and Pielou's (e) indices grouped bymouse life phases Pairwise Wilcoxon test was performed between incremental pairs of timepoints. Statistical significance is denoted with asterisks (*FDR-corrected $P<0.05$, **FDR-corrected $P<0.01$, ***FDR-corrected $P<$

590 denotes 'middle age', 'OD' denotes 'old' and 'VO' denotes 'very old'. PCoA plots showing 591 principal coordinates (PC) 1 and 2 of (f) Bray-Curtis dissimilarity and (g) weighted-UniFrac 
592 distance matrices. Percentage variation is shown in parenthesis. Beta-diversity measures were

593 based on an OTU table rarefied to 3,422 counts per sample.

594

595 Figure 2. Longitudinal changes in relative abundances of bacterial taxa. (a) A stacked plot 596 showing phylum groupings of major OTUs ( $\geq 0.5 \%)$. OTUs $<0.5 \%$ are grouped as “others". Each

597 horizontal black line denotes an OTU classified at 99\% similarity cut-off. (b) A line graph showing

598 the change in mean relative abundance (\%) of Firmicutes and Bacteroidetes over the 103 week

599 study. (c) Mean relative abundances of OTUs with the top 5 importance score using a random

600 forest regression method. Importance score in percentages are shown in parenthesis.

601 Actinobacteria, Cyanobacteria and Tenericutes are represented by fewer than 5 predictive OTUs.

602 Data points and error bars are the mean \pm standard error of the mean, respectively. Relative

603 abundances are based on an OTU table rarefied to 3,422 counts per sample. Taxonomic

604 assignments $>99 \%$ nucleotide identity for species and 95\%-99\% identity for genus level were

605 based on top BLASTn hits and $<95 \%$ nucleotide identity for family level were based on the SILVA

606 SSU database 132 release.

607

608 Figure 3. Normalized counts of 110 OTUs that have the highest mean over the 103-week-study.

609 Counts are based on a rarefied OTU table of 3,422 counts per sample. Asterisks indicate predicted

610 OTUs using the random forest regression method.

611

612 Figure 4. Host life-phase and age estimation based on fecal microbiota composition. (a)

613 SourceTracker based prediction of probabilities for each time-point to the five life phases. Symbols

614 in black outline show the timepoints for life phases used as 'source'. (b) Correlation of predicted 
615 age to actual mouse age. The Spearman correlation coefficient (rho), adjusted- $\mathrm{R}^{2}$ value of the

616 polynomial regression and thir $P$-values are shown.

617

618 Supplementary Figure S1. Survival plot of male C57BL/6J mice throughout the study. The red

619 line indicates when the mice arrived at the housing facility and when the first fecal samples were

620 collected. Longer tick marks on $\mathrm{x}$-axis indicate sampling time points.

621

622 Supplementary Figure S2. Longitudinal changes in alpha-diversity analysis of fecal microbiota 623 of 9- to 112-week-old C57BL/6J mice $(n=4-20)$. All alpha-diversity measures are based on an 624 OTU table rarefied to 3,422 counts per sample. 'MR' (weeks 9-12; $n=4-19$ ), 'MA' (weeks 17-22; $625 n=19$ ), 'MD' (weeks 43-60; $n=15-20$ ), 'OD' (weeks 82-104; $n=15-18$ ) and 'VO' (weeks 108$626112 ; n=12-13)$.

628 Supplementary Figure S3. Principal coordinate analysis (PCoA) plots based on beta-diversity 629 measures. PCoA plots showing PC1 relative to age of (c) Bray-Curtis dissimilarity and (d) 630 weighted-UniFrac distance matrices. Percentage variation is shown in parenthesis. Beta-diversity 631 measures were based on an OTU table rarefied to 3,422 counts per sample. OTUs were clustered 632 at $99 \%$ similarity cut-off.

634 Supplementary Figure S4. Longitudinal changes in mean relative abundance of less abundant 635 phyla (mean relative abundance $\leq 3 \%$ ). Relative abundances are based on an OTU table rarefied to 6363,422 counts per sample. 
638 Supplementary Figure S5. Normalized counts of predictive 28 OTUs not among the top 110

639 OTUs using a random forest regression method. Normalized counts are based on an OTU table

640 rarefied to 3,422 counts per sample. Taxonomic assignments $>99 \%$ nucleotide identity for species

641 and 95\%-99\% identity for genus level were based on top BLASTn hits and <95\% nucleotide

642 identity for family level were based on the SILVA SSU database 132 release.

644 Supplementary Figure S6. Beta-diversity measures and pairwise PERMANOVA test between

645 timepoints. The weighted-UniFrac distances of five timepoints of each life phase relative to the

646 other time-points (a). The five time-points arranged from top to bottom are 12 weeks old, 22 weeks

647 old, 56 weeks old, 91 weeks old and 108 weeks old. Red dot and red line denote FDR-corrected

$648 P$-values based on pairwise PERMANOVA test using 9,999 random permutations. An FDR-

649 corrected $P<0.05$ is considered statistically significantly different. Horizontal dotted line indicates

650 the FDR-corrected $P=0.05$ cut-off. A summary of total count of significantly different pairs of

651 microbiota across the study period relative to each timepoint for Bray-Curtis dissimilarity and

652 weighted UniFrac matrices (b). Dotted line indicates the line of best fit over the 26 timepoints for

653 each beta-diversity matrix. Black dots on Bray-Curtis plot denotes the points used as 'source' for

654 SourceTracker.

655 
a
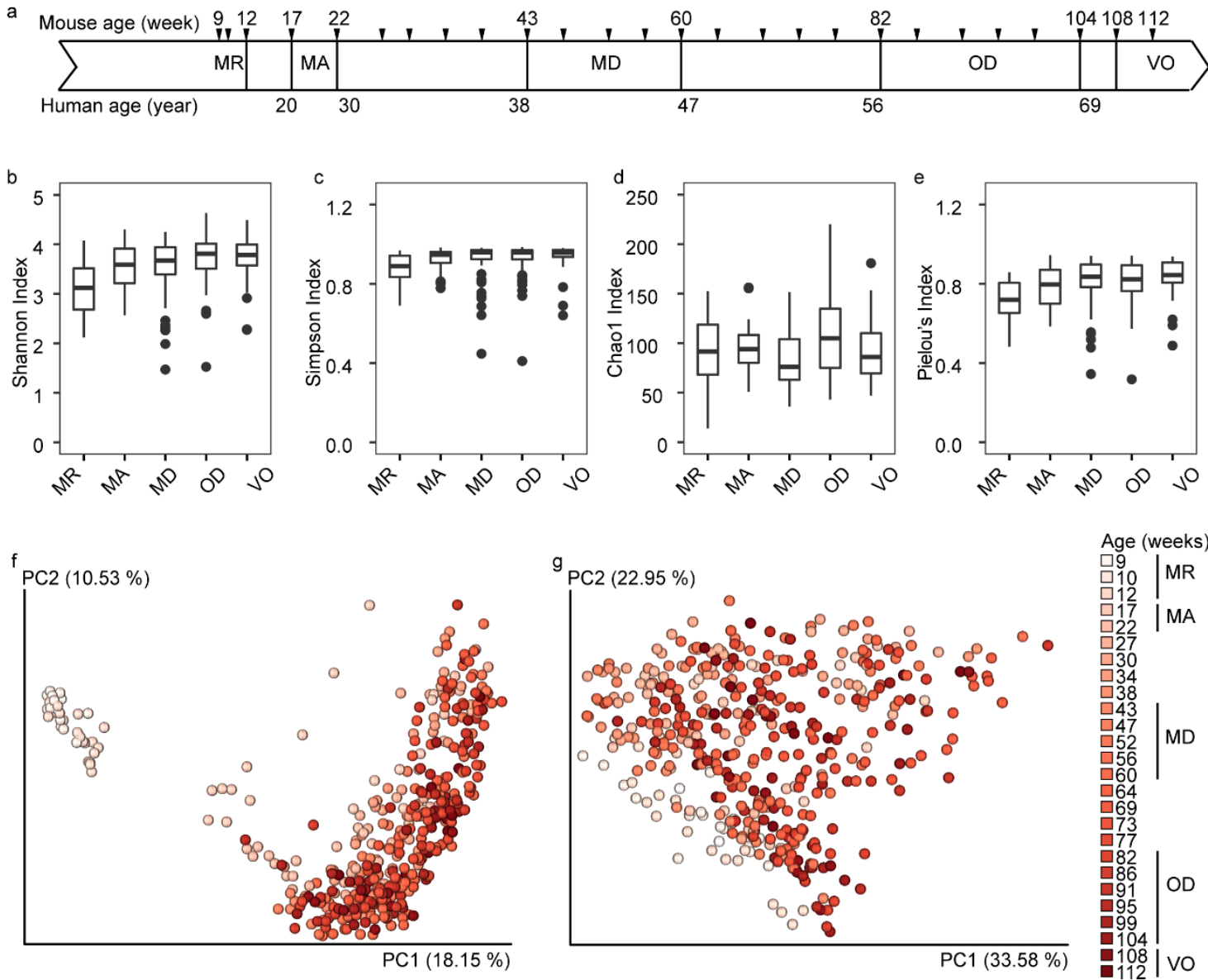

\section{Figure 1}



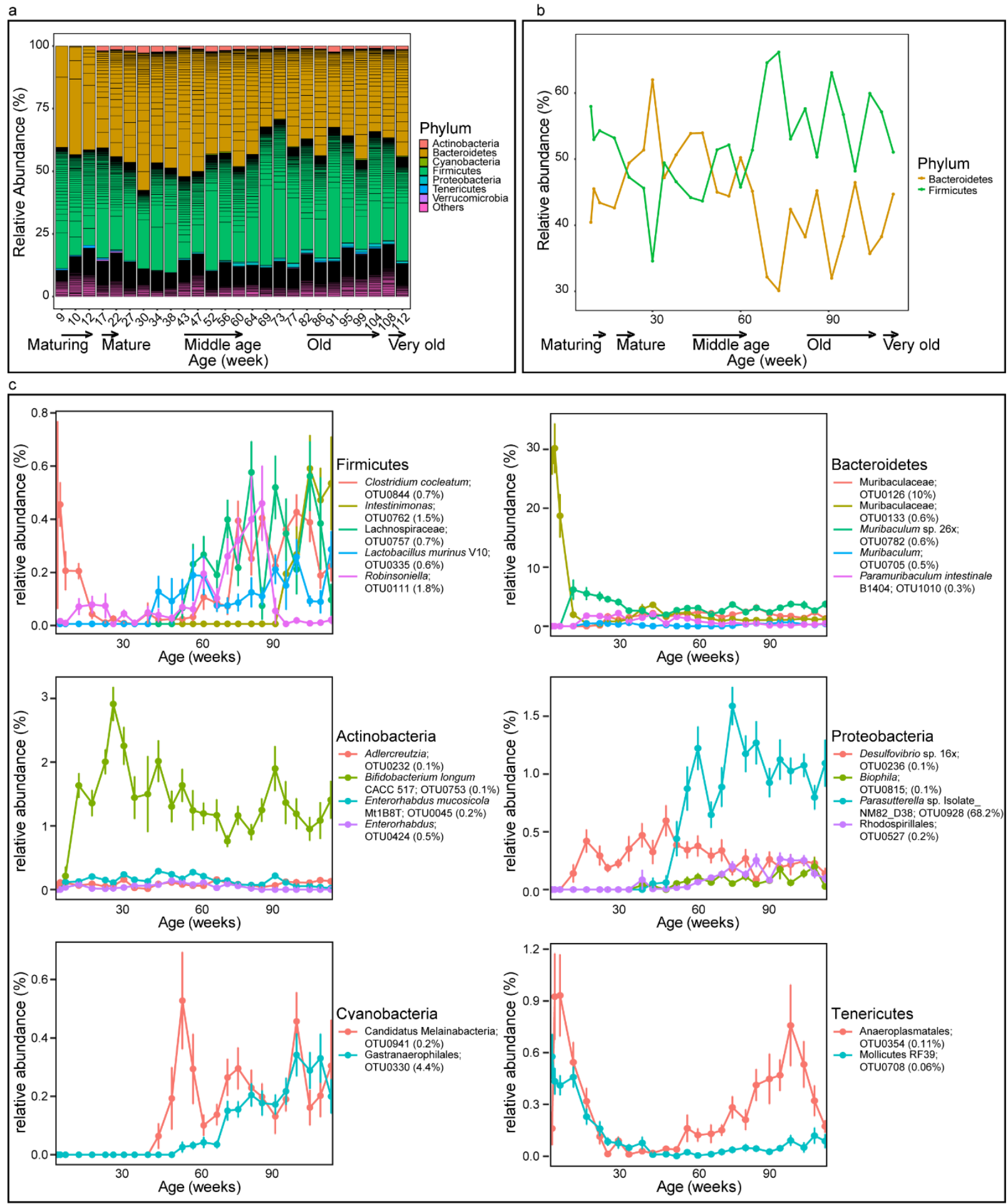
bioRxiv preprint doi: https://doi.org/10.1101/2020.12.04.412734; this version posted December 6, 2020. The copyright holder for this preprint (which was not certified by peer review) is the author/funder. All rights reserved. No reuse allowed without permission.

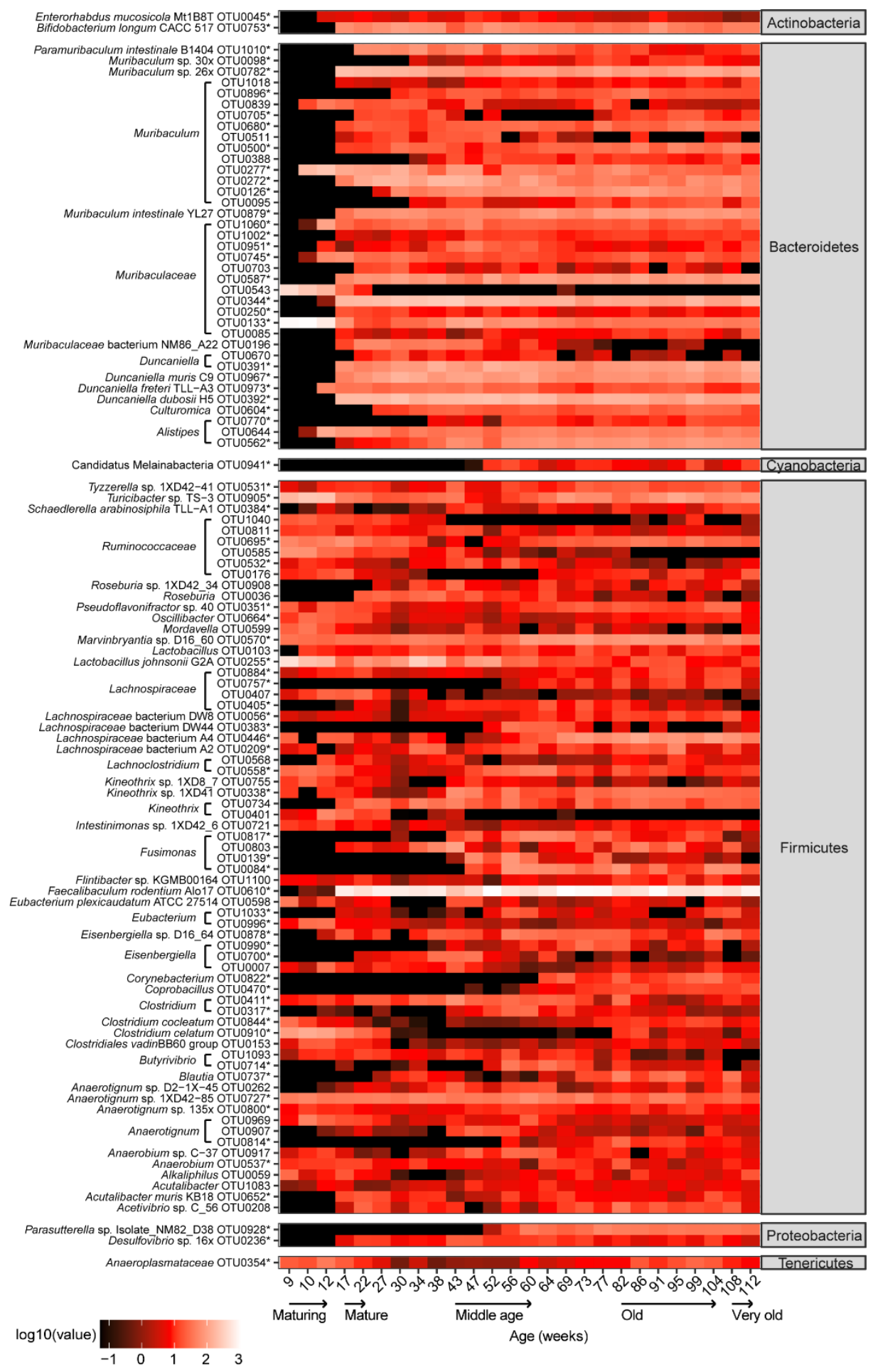

\section{Figure 3}




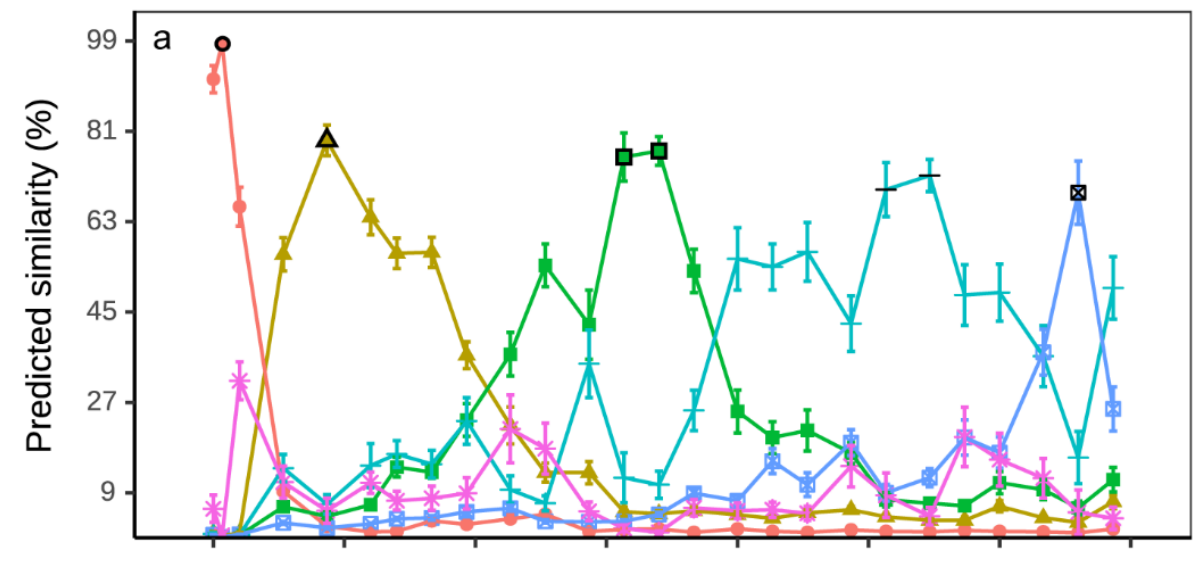

\section{Life-phase}

$\rightarrow$ Maturing

$\simeq$ Mature

$\rightarrow-$ Middle-age

+ Old

$\rightarrow$ Very old

* Unknown

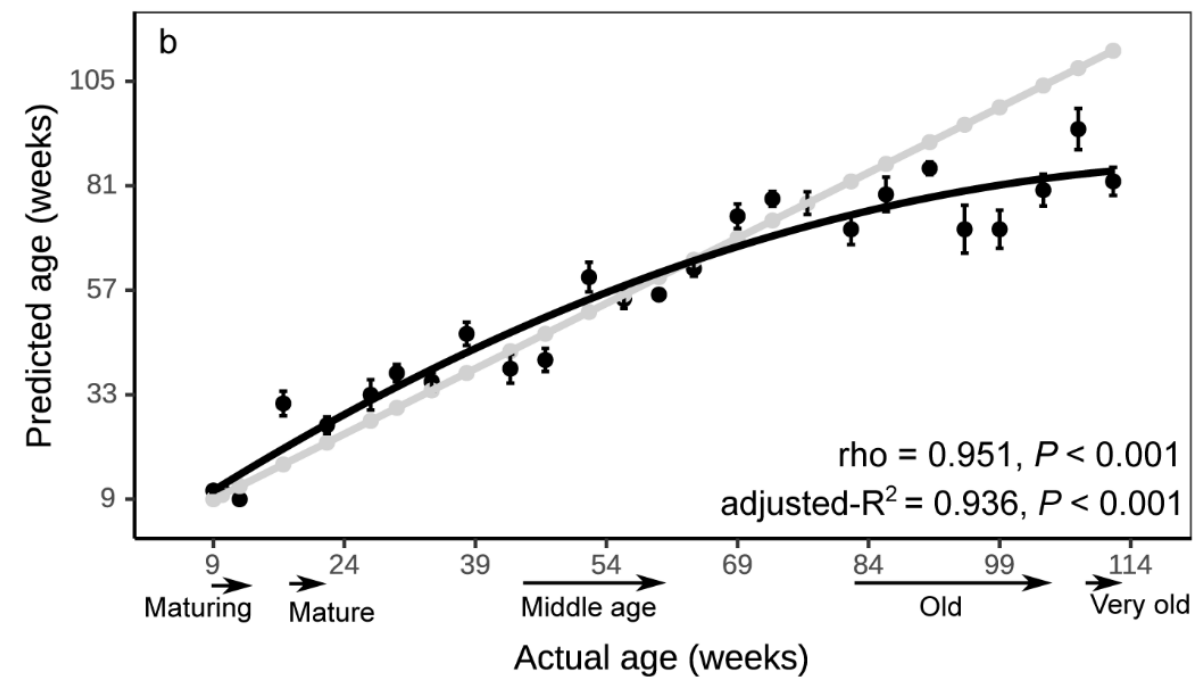

Actual age

Predicted age

664

665 Figure 4 